University of Wollongong

Research Online

Faculty of Engineering - Papers (Archive)

Faculty of Engineering and Information

Sciences

2005

\title{
The use of embedded sensors for the monitoring of adhesive joints in marine environments
}

\author{
Scott T. McGovern \\ University of Wollongong, scottmcg@uow.edu.au \\ Geoffrey M. Spinks \\ University of Wollongong, gspinks@uow.edu.au \\ G G. Wallace \\ University of Wollongong, gwallace@uow.edu.au
}

Follow this and additional works at: https://ro.uow.edu.au/engpapers

Part of the Engineering Commons

https://ro.uow.edu.au/engpapers/4247

\section{Recommended Citation}

McGovern, Scott T.; Spinks, Geoffrey M.; and Wallace, G G.: The use of embedded sensors for the monitoring of adhesive joints in marine environments 2005, 76-86.

https://ro.uow.edu.au/engpapers/4247

Research Online is the open access institutional repository for the University of Wollongong. For further information contact the UOW Library: research-pubs@uow.edu.au 


\title{
The use of embedded sensors for the monitoring of adhesive joints in marine environments
}

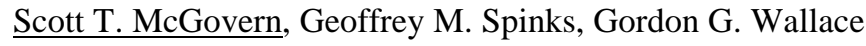 \\ ARC Centre for Nanostructured Materials \\ Intelligent Polymer Research Institute, University of Wollongong \\ Northfields Ave, Wollongong N.S.W 2522 \\ Ph: 0242213127 \\ Fax: 0242213114
}

\begin{abstract}
A copolymer incorporating polyaniline was used as a sensing medium in the construction of a resistance based humidity sensor. Aniline monomer was polymerised in the presence of poly (butyl acrylate / vinyl acetate) and a copolymer containing polyaniline emeraldine salt was obtained. The sensing medium was then developed by redissolving 1-2 $\mathrm{w} / \mathrm{w} \%$ of the resulting polymer residue in dichloromethane to produce a processable polymer blend solution. Some of this polymer residue was also de-doped in a solution of ammonia, and then washed with distilled water until the waste water had a neutral $\mathrm{pH}$. This residue was then redissolved at $1-2 \mathrm{w} / \mathrm{w} \%$ in dichloromethane to produce a second processable polymer blend this time containing polyaniline emeraldine base.

The final sensor design utilised $125 \mu \mathrm{m}$ polyester insulated platinum wire as conducting electrodes that were dip coated in the emeraldine salt copolymer solution and allowed to dry in a desiccator. The sensor was then dip-coated in a protective barrier layer of the emeraldine base copolymer to prevent over-oxidation and/or de-protonation of the emeraldine salt sensing medium under this coating. The sensors had an overall final thickness of less than $150 \mu \mathrm{m}$ and showed high sensitivity to humidity, low resistance, and good reversibility without hysteresis.

Sensors were monitored for 2-probe resistance changes when in contact with water. Calibration curves for each sensor were produced to convert the resistance reading to mass uptake of water. Individual sensors were embedded within Aluminium 5083 / Araldite 2015 adhesive joints to monitor mass uptake of water when exposed to marine environments. Correlations between mass uptake of water and joint strength were made.

There are various advantages of such a sensor design. Polymer based thin film humidity sensors have the advantage that the high processability of the material allows for simple fabrication of a range of geometries including smaller sensor designs. The ease of processing gives a low cost sensor, whilst the small size and good mechanical properties gives a robust sensor which has the flexibility to be able to be used in applications where dynamic stresses and strains are encountered. Such sensors may find uses in a number of areas including electronic textiles, food/ electronics packaging and corrosion detection.
\end{abstract}

Keywords: Polyaniline, Humidity, Sensor, Conducting Polymer, Adhesive, Fatigue, Marine

\section{INTRODUCTION}

The use of adhesives has been increasingly incorporated into the automotive and aerospace industries, due to the various structural and functional advantages when compared to traditional joining methods. Dissimilar metals may be joined with a reduced risk of cathodic corrosion. Lighter, thinner gauge metal sheets may be employed when joined with adhesives (where traditional welding methods determined the original gauge thickness), and this, along with the elimination of the weight of the welds themselves, can lead to a significant weight reduction in the structure. Adhesive joining methods tend to be faster than traditional welding, leading to cheaper labour costs during construction and repair. Better control of tolerances is achievable with adhesives due to the inherent distortion that can occur from the heat induced in welding processes. Even with these advantages, interfacial de-bonding of the adhesives is still a major concern with their use and has vastly limited their performance ${ }^{[1]}$. 
Degradation of adhesive joints can be linked to the diffusion or ingress of water into the joint, promoting hydration of the metal surface oxides and a loss in adhesive strength ${ }^{[1]}$. This effect is justifiably more pronounced in marine environments due to the highly humid and corrosive surroundings and to date there has been little or no use of adhesives in marine applications. As such, the ability to monitor the extent that water ingresses into an epoxy adhesive can be a useful tool to determine the strength and condition of the adhesive joint at any point in time.

To be able to accurately measure water ingress in adhesive joints it has been proposed to embed small sensors within the adhesive so that a continual monitoring of the localised water content may be achieved. For this to be successful, the sensor must be small enough so that the sensor itself does not harm the joint strength in any way yet still be stable and accurate. If the sensors could be made so that they were low cost, then an affordable yet complex array of multiple sensors could be used such that a virtual map of the water ingress into the structure could be seen at any time, and localisation of potential hazard points could be achieved.

Various studies have been conducted into novel materials that can be used to detect humidity. Some studies have concentrated upon ceramic materials where the change in impedance or dielectric properties of the system were used to detect the ingress of water ${ }^{[2-4]}$. Other systems incorporate humidity sensing polymers ${ }^{[5-10]}$ where either dielectric, capacitive or resistance based parameters have been found to change with water ingress. Each system in its own right measures the change in humidity (with consideration taken to hysteresis, linearity and reproducibility) but problems with overall size and processability of the sensing material can restrict applications in certain areas.

Ceramic humidity sensors based on $\mathrm{ZnO}, \mathrm{TiO}_{2}$ and $\mathrm{SiO}_{2}$ films have been developed that show highly sensitive, reversible, linear responses ${ }^{[2-4]}$. Processability of such designs however can be somewhat tedious requiring high temperatures and a multi layered construction to the sensor substrate. The use of sol-gels has improved this processability ${ }^{[11-15]}$ but most designs still require a glass or quartz crystal substrate which can restrict the degree of miniaturisation.

Much work has also been undertaken into the humidity sensing properties of conducting polymers. It is known that water increases the electrical conductivity of polyaniline through an increase in the interchain electron transfer ${ }^{[16-19]}$ and / or by increasing the mobility of dopant ions ${ }^{[20]}$. Conductivity increases of several orders of magnitude in the presence of water have been recorded with linear and reversible responses ${ }^{[19-21]}$.

Polymer based thin film humidity sensors have the advantage that the high processability of the material allows for simple fabrication of a range of geometries including smaller sensor designs. The ease of processing gives a low cost sensor, whilst the small size and good mechanical properties give a robust flexible sensor that is able to be used in applications where dynamic stresses and strains are encountered. With these advantages in mind, the aim of this study was to design, fabricate and evaluate humidity sensors using processable polyaniline blends as the sensing medium. In particular, the sensors have been assessed for linearity, sensitivity, bulk resistance, response time, temperature response and suitability for detection within a curing epoxy adhesive.

\section{METHODOLOGY}

\subsection{Sensor construction and calibration}

\subsubsection{Sensor substrate construction}

Sensor electrodes were made from $125 \mu \mathrm{m}$ platinum wire that was insulated with a $14 \mu \mathrm{m}$ polyester coating, as supplied from Goodfellow (UK). A $7 \mathrm{~cm}$ section of wire was cut, then folded in half and its ends were twitched together to create two parallel wires electrodes connected at one end by a loop of bare platinum. The insulation coating from both ends was removed by scorching with a hot Bunsen burner flame. The parallel wire sections were glued together in a flat orientation with cross-linked poly vinyl alcohol (PVA) and left to dry before the platinum wire loop was cut. The final substrate was checked to ensure that its thickness was no more than $150 \mu \mathrm{m}$ and the spacing between electrode ends was less than $500 \mu \mathrm{m}$. A typical finished sensor substrate can be seen in Figure 1). 
a)

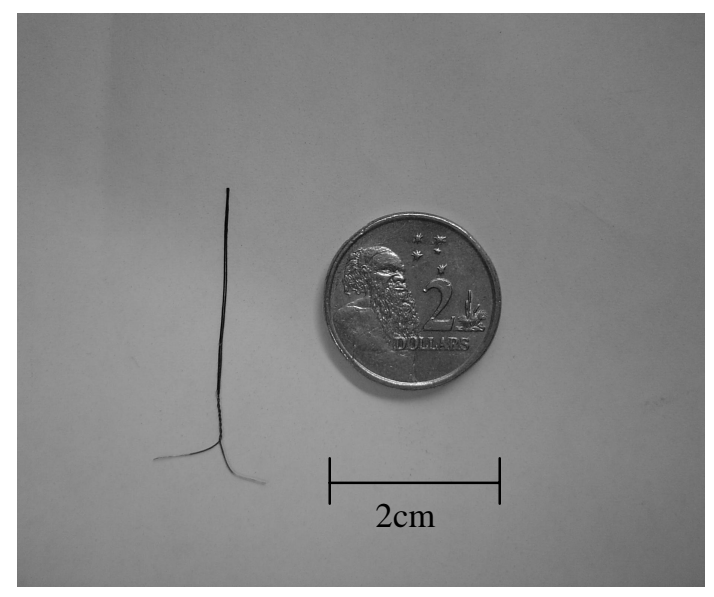

b)

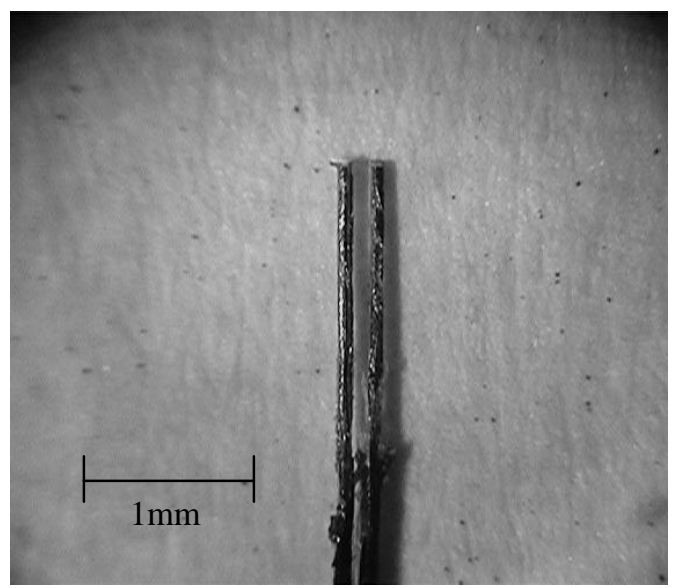

Figure 1) (a) Finished sensor substrate ready for coating, and (b) as seen at higher magnification showing bared insulation and gap of approx. $250 \mu \mathrm{m}$.

\subsubsection{Polymer Blends}

A processable polymer was made by the chemical polymerisation of aniline monomer to emeraldine salt (ES) with ammonium persulphate, in an emulsion of poly (butyl acrylate-co-vinyl acetate) $\mathrm{P}(\mathrm{BuA}-\mathrm{VAc})$ copolymer $(40 \mathrm{w} / \mathrm{w} \%)$ ${ }^{[22]}$. The $\mathrm{P}(\mathrm{BuA}-\mathrm{VAc})$ copolymer (Scheme I) was synthesised according to previously reported methods ${ }^{[23,24]}$ and the final polymerisation took place with camphor sulphonic acid (HCSA) being used in excess as the dopant. The end product was purified with a de-ionised water wash prior to centrifuging and the resulting solid was dried to a powder. The PAn percentage was determined from elemental analysis to be $15 \mathrm{w} / \mathrm{w} \%$ in the developed PAn/ P(BuA-VAc) blend. Some of the resulting powder was washed in ammonia solution to de-dope the PAn to emeraldine base (EB) (Scheme 1). This residue was then washed with de-ionised water until a neutral $\mathrm{pH}$ was realised with the waste water, and this was then also dried to a powder. The two polymers (ES and EB) were separately redissolved in $\mathrm{CH}_{2} \mathrm{Cl}_{2}$ solvent at $1-2 \%$ by weight to create dark green and dark blue liquids respectively, which could be readily stored in an easily processable form.

\subsubsection{Development of the sensors}

The sensor substrate was dip-coated with the PAn ES blend that was to be used as the sensing material and allowed to dry completely in a desiccator. After drying a second protective layer of the PAn EB was applied to the surface by dip coating. This layer was applied to stabilise the ES sensing material once embedded in the high $\mathrm{pH}$ curing epoxy and protect it from the possibility of de-doping, which would lead to a loss in conductivity and a change in the humidity response.

With this overall design the width of the conducting electrodes was the main limiting factor to the overall size and final sensor resistance. The width was kept to a minimum by aligning the electrodes under a microscope ensuring that no short circuits were present using a standard multimeter. The width was thus kept to around $500 \mu \mathrm{m}$ or less before the process of polymer dip-coating.

The other factor that determined the sensor resistance was the 'bridging thickness' of the conducting polymer between the electrodes (Figure 2).

The ES bridging thickness was examined under a microscope and was estimated to have a thickness of no less than $30 \mu \mathrm{m}$ and the total bridging thickness (ES with the EB outside coating) was estimated to be no less than $120 \mu \mathrm{m}$. 


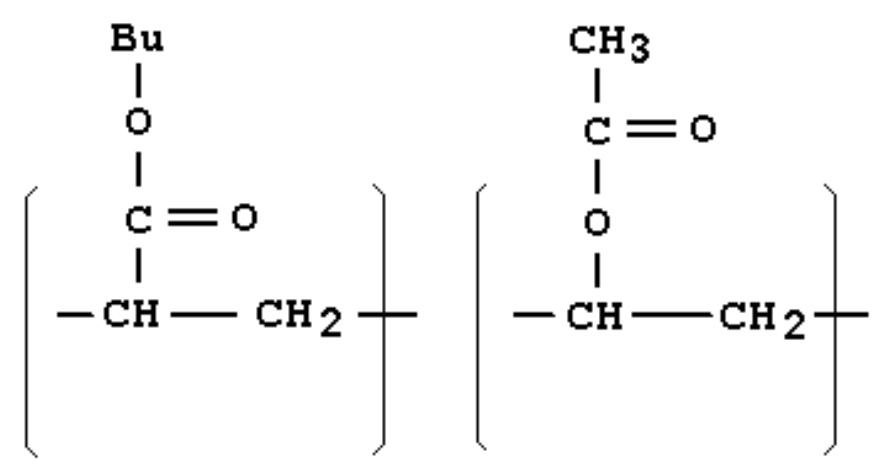

$\mathbf{n}$

m

poly(Butyl Acrylate -co- Vinyl Acetate)<smiles>CNc1ccc(N=[W]c2ccc(Nc3ccc(Nc4ccc(C)cc4)cc3)cc2)cc1</smiles>

polyaniline (emeraldine salt)<smiles>CN=C(NC)c1ccc(Nc2ccc(Nc3ccc(Nc4ccc(C)cc4)cc3)cc2)cc1</smiles>

polyaniline (emeraldine base)

Scheme I)

\subsubsection{Calibration of the sensors}

An Agilent 34401A Multimeter was used to measure resistances across the sensors when placed in chambers of varying humidity. The relative humidities $(\mathrm{RH})$ in each chamber were created using saturated salt solutions of $\mathrm{LiCl}, \mathrm{MgCl}_{2}$, $\mathrm{Ca}\left(\mathrm{NO}_{3}\right)_{2}$ and $\mathrm{NaNO}_{3}$ which correspond to relative humidities of $11,33,51$, and $74 \%$ respectively ${ }^{[25]}$. A chamber containing silica gel was also used and was found to give a 3\% RH after checking with a commercial humidity sensor. The resistance for each humidity level was measured after equilibration in each of the humidity chambers to produce an overall bulk resistance calibration curve for each sensor. For ease in comparison from one sensor to the next, the resistance reading at $74 \% \mathrm{RH}$ for each sensor was set as a baseline and the $\%$ change in resistance was then calculated for each RH and second calibration curve produced. This second curve gave the equilibrium humidity sensing characteristics of the PAn ES material eliminating factors such as spatial constraints. 
In particular, the sensors were assessed for linearity, sensitivity, bulk resistance, response time and temperature response.

a)

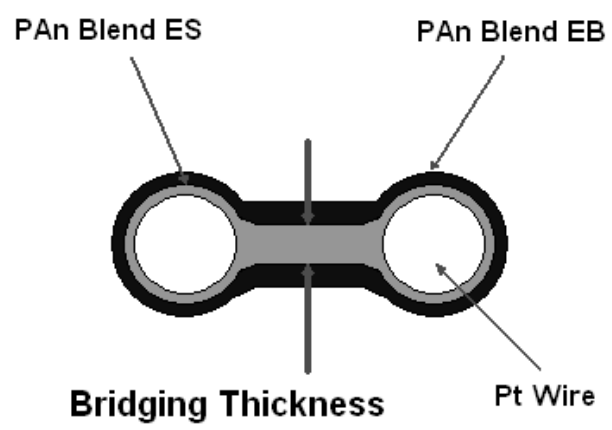

b)

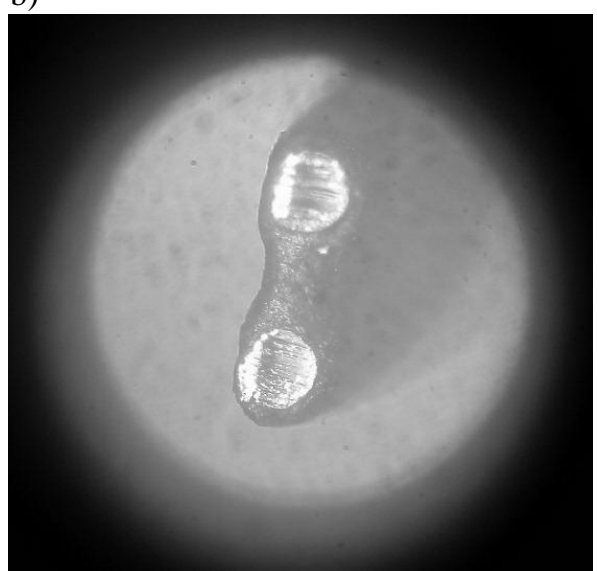

Figure 2) a) Schematic of the cross-sectional view across the conducting polymer end of the sensor, b) A photo showing the same cross-sectional view.

To convert sensor readings to w/w\% water in the sensor, films of PAn BuA-co-VAc ES were first cast, then dried in $3 \% \mathrm{RH}$ and the equilibrium mass was measured. These films were then placed in chambers of ascending RH and the equilibrium masses measured. Mass uptake of water in the PAn films for increasing RH was calculated by comparing the equilibrium masses for each RH to the dried films. A curve was produced so that RH values for the sensor could be converted to w/w\% water in the PAn - a more meaningful figure for embedded sensors. This curve may be seen in Figure 3).

\subsection{Testing the Sensors}

\subsubsection{Construction of adhesive joints}

Adhesive joints were constructed from Aluminium 5083 metal strips joined with Araldite 2015 epoxy adhesive in a lap shear configuration. $2 \mathrm{~mm}$ thick aluminium 5083 plating was cut into strips $15 \mathrm{~mm}$ wide and $120 \mathrm{~mm}$ long and joined together with an overlap of $12 \mathrm{~mm}$. A solvent-wipe pre-treatment of the surfaces with acetone was conducted to remove residual oils and dirt. Strips were held together with a metal clip during curing of the adhesive and spew fillets were removed with a paint scraper blade prior to immersion in salt water spray.

\subsubsection{Salt water spray chamber and strength testing}

A chamber was setup to spray a $5 \mathrm{w} / \mathrm{w} \% \mathrm{NaCl}$ salt water solution onto samples being tested. The temperature of the sprayed water was kept at a constant $26+/-2^{\circ} \mathrm{C}$ during the lifetime of the test and adhesive joints with sensors were compared with joints without sensors for varying times of immersion. Tensile strength of the joints was measured on the samples using an Instron 4302 operating at a speed of $1 \mathrm{~mm} / \mathrm{min}$ extension after removal from the test apparatus after removal from the test apparatus. 


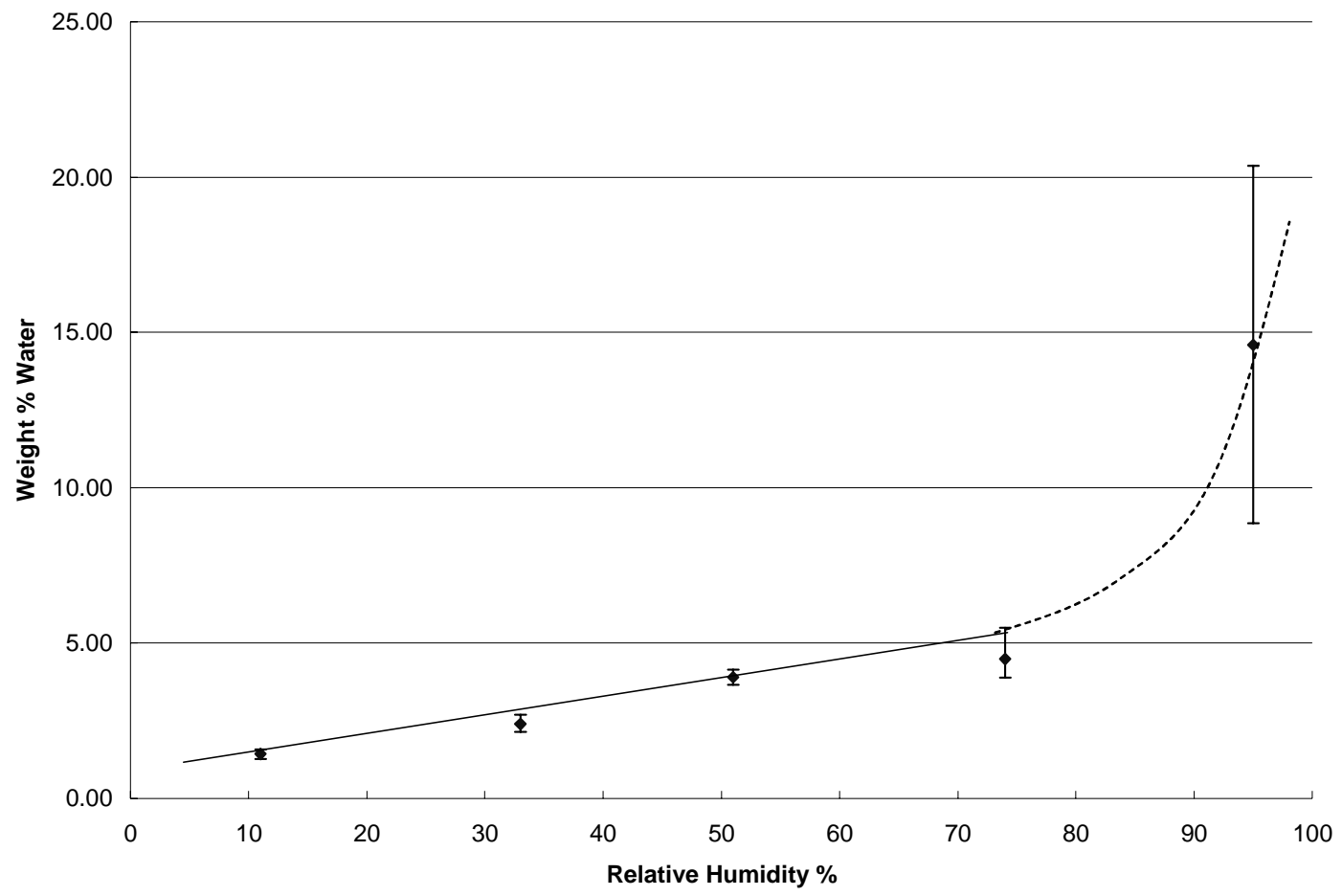

Figure 3) Calibration curve for converting RH to weight $\%$ water in PAn sensors. The dashed line indicates a region where the sensor accuracy is uncertain.

\section{RESULTS AND DISCUSSION}

\subsection{Sensor Calibration and Response}

\subsubsection{Linearity/ Sensitivity}

Calibration curves for the $15 \mathrm{w} / \mathrm{w} \%$ PAn ES blend with EB protective coating responses were found to be almost exponential and from $11-74 \% \mathrm{RH}$. A high sensitivity was recorded with over a $300 \%$ resistance change from $11-$ $74 \%$ RH.

The \%change in resistance calibration curves were repeated many times and the average values can be seen in Figure 4) along with the standard deviation at each RH level.

\subsubsection{Effect of Electrode Separation}

The final spacing of the conducting electrodes was found to be the main factor in determining the resulting sensor resistance. With electrodes that were less than $1 \mathrm{~mm}$ apart, each blend gave sensors that showed bulk resistance measurements (at 3\% RH) less than $10 \mathrm{k} \Omega$, making it an appropriate transducer for practical applications. 


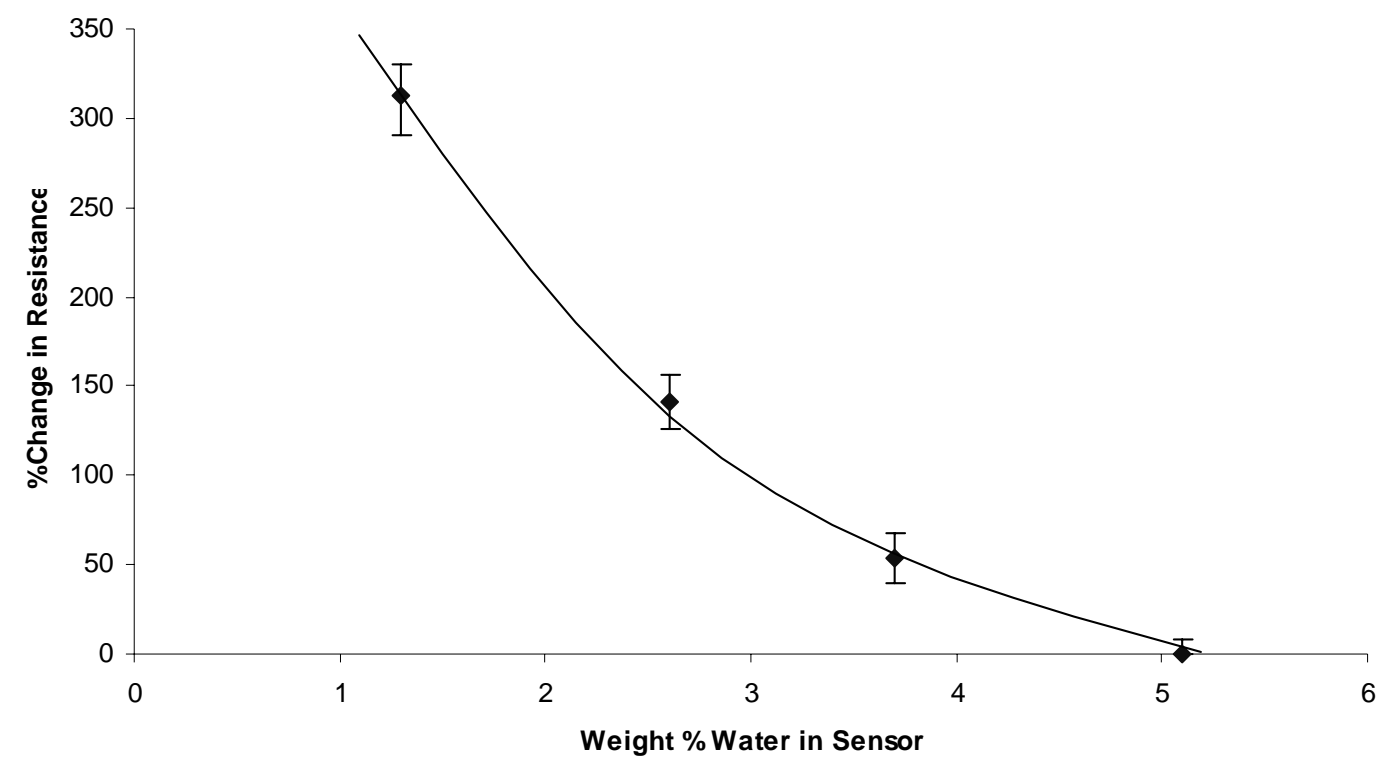

Figure 4) \%Change in resistance calibration curve for PAn $\mathrm{P}(\mathrm{BuA}-\mathrm{co}-\mathrm{VAc})$ ES sensors with EB protective coating over varying relative humidity and hence mass uptake water

\subsubsection{Response Time}

The rate of water ingress into or out of the polymer was seen to be dependant on the differential between the current concentration of water in the sensor and the concentration of water in the surrounding atmosphere. As could be expected, the larger this differential, the longer the time needed to reach equilibrium in the sensor and the times required to reach equilibrium for humidifying stages were much faster than that for desiccating stages. The sensors took approximately 6 to 7 hours to reach $90 \%$ of the equilibrium resistance on humidifying and 30 hours was required to reach $90 \%$ of the equilibrium resistance on desiccating.

In general, the sensors required several hours to equilibrate after a step change in RH. It is expected that producing thinner coatings over a substrate with a smaller gap between platinum wire electrodes could further reduce the sensor response time.

\subsubsection{Temperature Response}

The sensors have been found to be slightly responsive to temperature as shown by the calibration curve from temperature changes of 5 to $60^{\circ} \mathrm{C}$ in constant $3 \% \mathrm{RH}$, seen in Figure 5). Similar resistance decreases have been reported with increases in temperature ${ }^{[26-28]}$ and likewise have been attributed to an increased mobility and movement of either conducting electrons or un-doped dopants.

\subsection{Sensors performance in service}

\subsubsection{Sensor performance within adhesive joints}

Sensor response was monitored over varying exposure times of adhesive joints to salt water spray. A standard Q test was applied to results with a $90 \%$ confidence interval to generate the graph seen in Figure 6).

The sensors were placed within the adhesive joints along the centre of the bondline so that the sensor itself would show minimal interference during later strength testing. As such the time lag between initiation of the test and when water had started to diffuse into the centre of the adhesive was $15 \mathrm{hr}$. At this point the sensor monitored the moisture ingress and it was seen that the adhesive was fully saturated at about 35-40 hrs. A typical sensor response may be seen in Figure 6). 


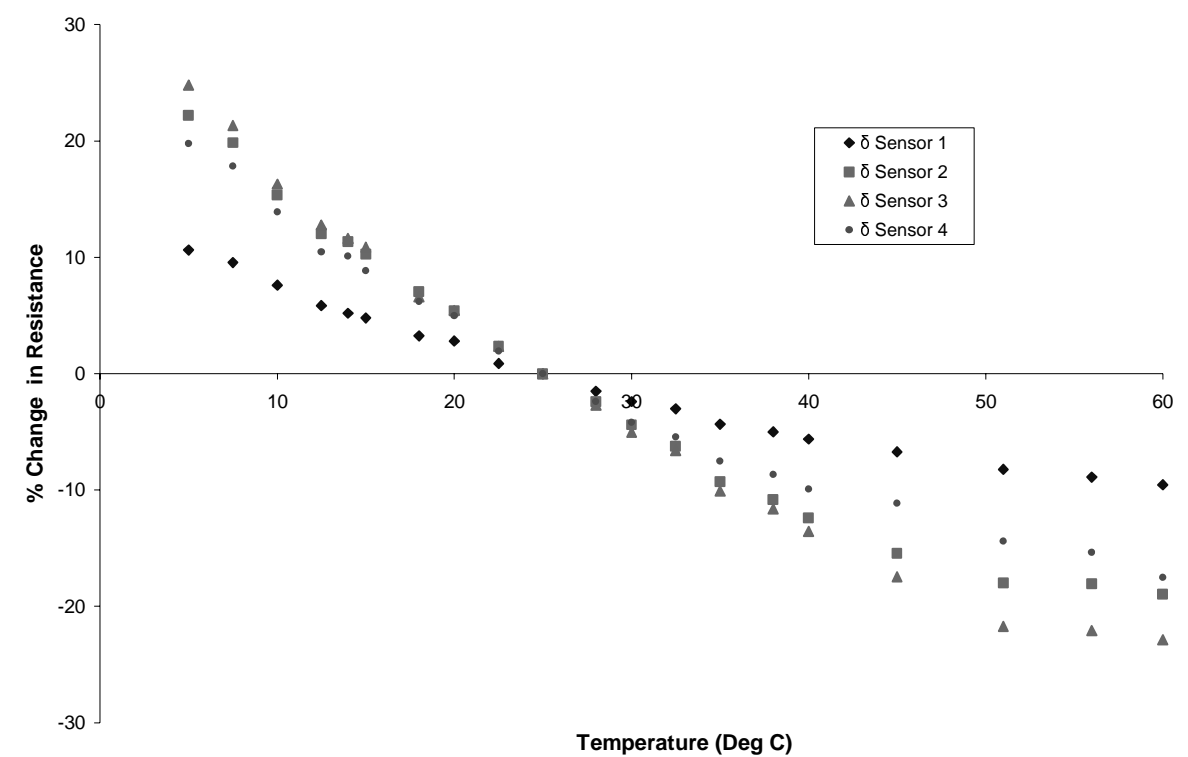

Figure 5) Typical temperature calibration curves for four different 15 w/w\% PAn P(BuA-co-VAc) ES sensors in a constant $3 \%$ RH

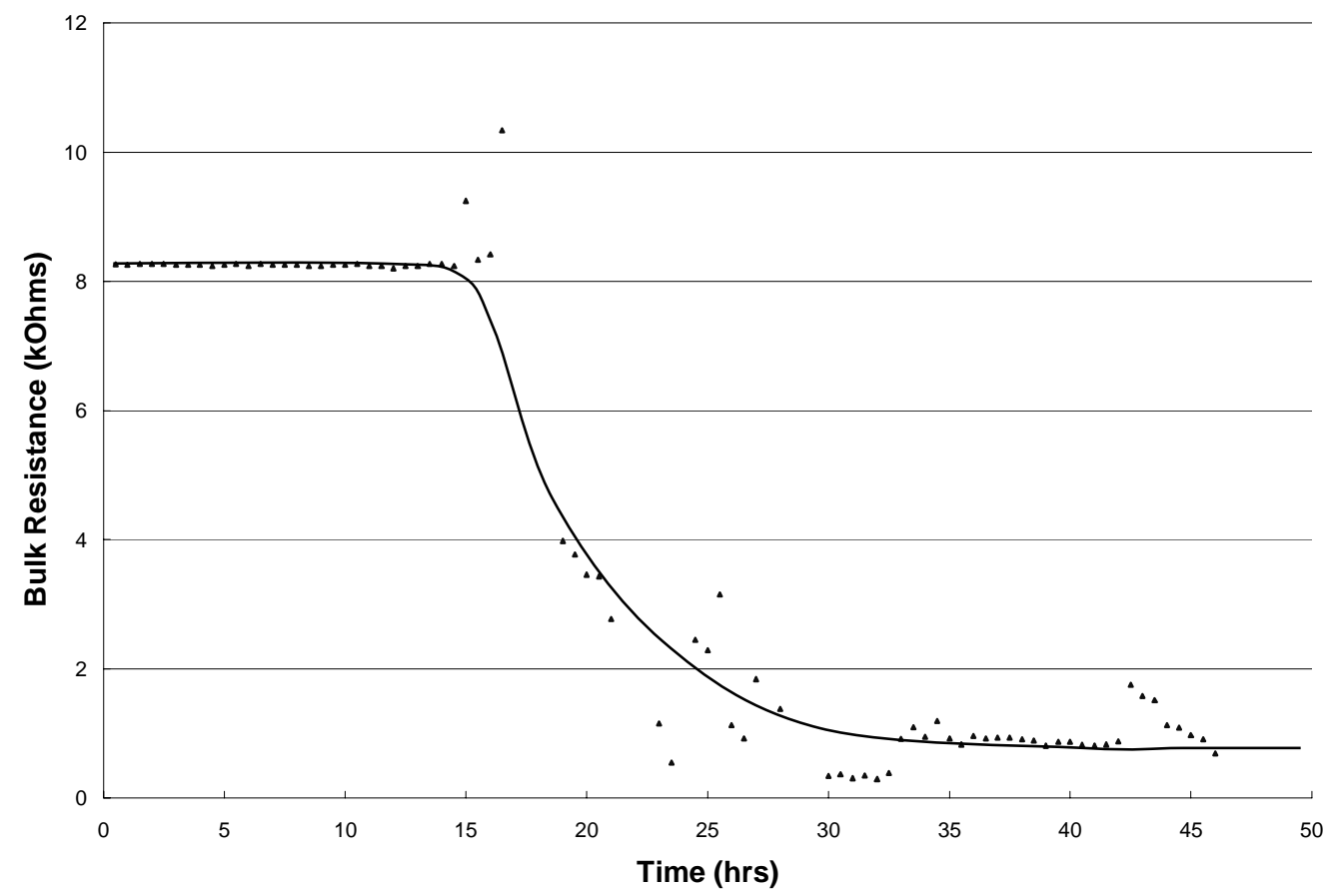

Figure 6) Typical embedded sensor response within Araldite 2015 Aluminium 5083 adhesive joints upon exposure to constant temperature salt water spray. 


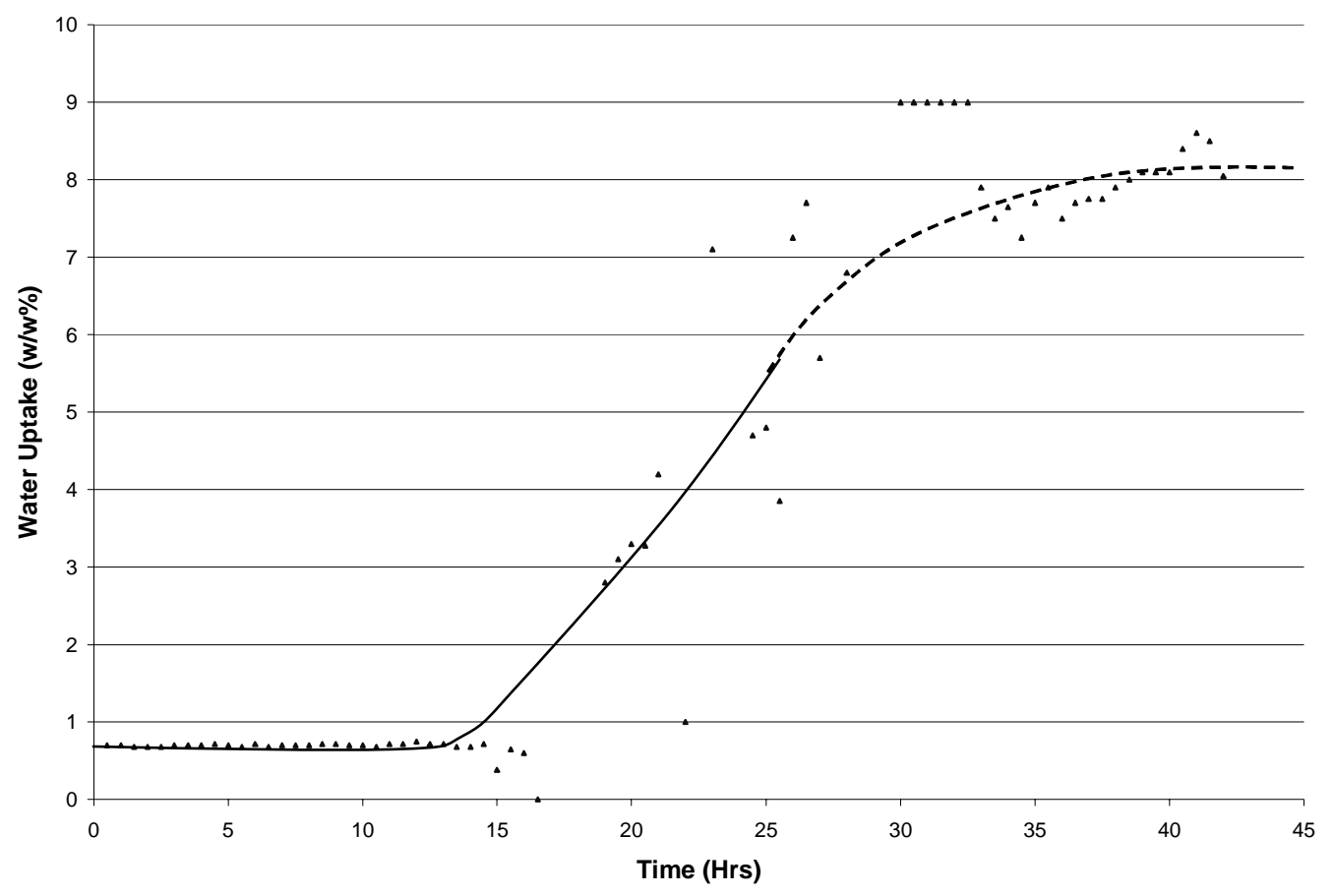

Figure 7) Conversion of embedded sensor resistance response within Araldite 2015 Aluminium 5083 adhesive joints to mass uptake of water in the sensing material upon exposure to constant temperature salt water spray over varying time. The dashed line indicates a region where the sensor accuracy is uncertain.

The sensor resistance reading was converted to a reading of mass uptake of water and this graph may be seen in Figure 7). It was seen that the adhesive was fully saturated by about $40 \mathrm{hrs}$ having in excess of $5 \mathrm{w} / \mathrm{w} \%$ water. Further work is required to accurately determine the water content above this level.

It was seen that the sensor has a quicker response time in soaking water $(90 \%$ saturated within $6-7 \mathrm{hrs})$ when compared to the Araldite adhesive (90\% saturated in at least $15 \mathrm{hrs}$ ), so it can be expected that the sensor will be able to monitor accurately the water content in the surrounding adhesive as it changes. It should also be noted that the sensor has a very thin coating of PAn consisting of extremely low mass when compared to the bulk adhesive, so even though the PAn has a higher affinity for water, the change in diffusion characteristics (if any) would likely be minimal due to the embedded sensor.

This sensor can be seen to be a useful tool in monitoring the rate of water ingress into the Araldite 2015, though to be able to accurately quantify the water content in the adhesive further calibration is needed of sensor response vs. w/w\% water in Araldite 2015.

\subsubsection{Adhesive joint strength}

A graph of tensile strength of Araldite 2015 Aluminium 5083 adhesive joints for varying exposure times to salt water spray was produced with and without embedded sensors and may be seen in Figure 8). It can be seen that the tensile strength of the adhesive joints initially increases after $24 \mathrm{hrs}$ exposure to salt water spray and this effect can be attributed to a plasticisation of the adhesive where the toughness of the adhesive increases would have appeared to have increased somewhat. The $\mathrm{w} / \mathrm{w} \%$ water in the sensing material was approximately $5 \%$ at this point.

After 24 hrs only a slight decrease in tensile strength is seen counteracting the increase due to plasticisation of the adhesive, even up to 10 days exposure, and this was attributed to an oxidation of the aluminium bonding surface and salt water attack on the oxide layer. Each of the adhesive joints showed adhesive failure at the metal surface and with different metal surface pre-treatments it would be expected to see a change from cohesive to adhesive failure with the 
onset of corrosion at the metal surface. It should be noted that even though these results are preliminary, further salt water attack is expected at longer exposure times. Further tests into varying metal surface pre-treatment prior to bonding as well as longer exposure times are in progress.

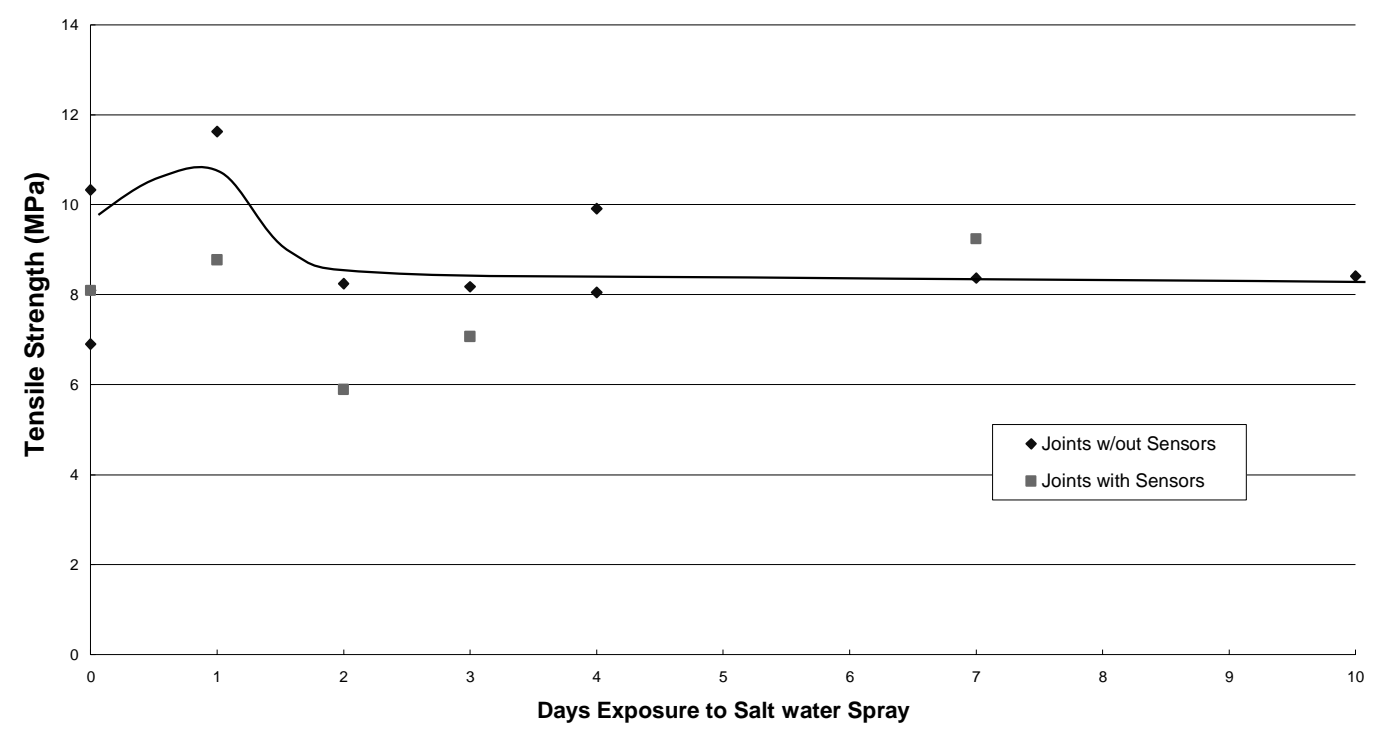

Figure 8) Tensile strength of Araldite 2015 Aluminium 5083 adhesive joints with a solvent wipe pre-treatment after being exposed to a constant temperature salt water spray for varying lengths in time

\section{CONCLUSIONS}

Micro humidity sensors were constructed from polyester insulated platinum wire substrate to have a thickness of no more than $150 \mu \mathrm{m}$. Two processable polyaniline blends for substrate dip-coating were developed both with a butyl acrylate / vinyl acetate copolymer $(\mathrm{P}(\mathrm{BuA} / \mathrm{VAc}))$, each with $15 \mathrm{w} / \mathrm{w} \% \mathrm{PAn}$ in the final sensing material. One of the blends consisted of PAn in the conducting emeraldine salt ES form and the other with PAn in the non-conducting emeraldine base form. The sensor consisted of the substrate being coated in the PAn ES as the sensing material, utilising a further PAn EB coating as a protective barrier layer against ES deprotonation. The sensors were investigated for their ability to sense atmospheric humidity and water content in Araldite 2015 Aluminium 5083 adhesive joints with an Acetone solvent wipe pre-treatment that were exposed to salt water spray. The sensors showed consistent and measurable responses with a slightly exponential shape. High sensitivities were seen, representing over a $300 \%$ resistance change from $11-74 \% \mathrm{RH}$.

Adhesive joints were shown to have a small increase in tensile strength after 24 hrs exposure corresponding to $5 \mathrm{w} / \mathrm{w} \%$ water in the sensing material. The adhesive joints were seen to be fully saturated after $35-40$ hrs though only a small drop in tensile strength was seen even up to 10 days exposure to salt water spray. The sensor has been shown to be a useful tool in monitoring the rate of water ingress into Araldite 2015, but further work is needed to be able to quantify levels of water in the adhesive.

Further work is in progress into developing calibration curves for mass uptake of water in the Araldite 2015 adhesive vs. sensor response so that water content in the adhesive can be properly quantified. Other tests will involve the varying of the metal surface pre-treatment types and the subjecting of adhesive joints for longer exposure times. Samples will also be subjected to a $1 \mathrm{~Hz}$ fatigue cycle with varying loads to examine the change in water uptake as micro cracks form during the fatigue process. 


\section{ACKNOWLEDGEMENTS}

The authors like to thank Reza Mirmosheni and Magnus Gustavson for their help with the synthesis of the conducting polymer blends. They would also like to thank Huntsman for their financial assistance with the project.

\section{REFERENCES}

[1] O. O. Lunder, B;Nisancioglu,K, Pretreatment of AA6060 auminium alloy for adhesive bonding, Int. Journal of Adhesion and Adhesives 22, (2002) pp. 143-150

[2] M. Sato and T. Yamamoto, Sensitivity of phase velocity of a composite ZnO plate to humidity, Sensors and Actuators B: Chemical, 13, (1993) pp. 96-99

[3] N. Ichinose, Humidity sensitive characteristics of the MO---WO3 ( $\mathrm{M}=\mathrm{Mg}, \mathrm{Zn}, \mathrm{Ni}, \mathrm{Mn}$ ) system, Sensors and Actuators B: Chemical, 13, (1993) pp. 100-103

[4] E. Traversa, A. Bearzotti, M. Miyayama and H. Yanagida, Study of the conduction mechanism of La2CuO4--ZnO heterocontacts at different relative humidities, Sensors and Actuators B: Chemical, 25, (1995) pp. 714-718

[5] Y. Sakai, Y. Sadaoka and M. Matsuguchi, Humidity sensors based on polymer thin films, Sensors and Actuators B: Chemical, 35, (1996) pp. 85-90

[6] Y. Sakai, Humidity sensors using chemically modified polymeric materials, Sensors and Actuators B: Chemical, 13, (1993) pp. 82-85

[7] P. R. Story, D. W. Galipeau and R. D. Mileham, A study of low-cost sensors for measuring low relative humidity, Sensors and Actuators B: Chemical, 25, (1995) pp. 681-685

[8] C.-D. Feng, H. Wang, S.-L. Sun, C. U. Segre and J. R. Stetter, Comparison of conductometric humidity-sensing polymers, Sensors and Actuators B: Chemical, 40, (1997) pp. 211-216

[9] T. Kuroiwa, T. Hayashi, A. Ito, M. Matsuguchi, Y. Sadaoka and Y. Sakai, A thin film polyimide based capacitive type relative humidity sensor, Sensors and Actuators B: Chemical, 13, (1993) pp. 89-91

[10] C. Roman, O. Bodea, N. Prodan, A. Levi, E. Cordos and I. Manoviciu, A capacitive-type humidity sensor using crosslinked poly(methyl methacrylate-co-(2 hydroxypropyl)-methacrylate), Sensors and Actuators B: Chemical, 25, (1995) pp. 710-713

[11] G. Vaivars, J. Pitkevics and A. Lusis, Sol-gel produced humidity sensor, Sensors and Actuators B: Chemical, 13, (1993) pp. 111-113

[12] H.-T. Sun, Z.-T. Cheng, X. Yao and W. Wlodarski, Humidity sensor using sol--gel-derived silica coating on quartz crystal, Sensors and Actuators B: Chemical, 13, (1993) pp. 107-110

[13] C.-D. Feng, S.-L. Sun, H. Wang, C. U. Segre and J. R. Stetter, Humidity sensing properties of Nafion and sol-gel derived SiO2/Nafion composite thin films, Sensors and Actuators B: Chemical, 40, (1997) pp. 217-222

[14] G. Montesperelli, A. Pumo, E. Traversa, Gusmano, A. Bearzotti, A. Montenero and G. Gnappi, Sol--gel processed TiO2-based thin films as innovative humidity sensors, Sensors and Actuators B: Chemical, 25, (1995) pp. 705-709

[15] J. Lin, M. Heurich and E. Obermeier, Manufacture and examination of various spin-on glass films with respect to their humidity-sensitive properties, Sensors and Actuators B: Chemical, 13, (1993) pp. 104-106

[16] M. J. Winokur and B. R. Mattes, Structural Studies of Halogen Acid Doped Polyaniline and the Role of Water Hydration., Macromolecules, 31, (1998) pp. 8183 - 8191

[17] O. N. Timofeeva, B. Z. Lubentsov, Y. Z. Sudakova, D. N. Chernyshov and M. L. Khidekel', Conducting polymer interaction with gaseous substances I. Water, Synthetic Metals, 40, (1991) pp. 111-116

[18] B. Lubentsov, O. Timofeeva, S. Saratovskikh, V. Krinichnyi, A. Pelekh, V. Dmitrenko and M. Khidekel', The study of conducting polymer interaction with gaseous substances IV. The water content influence on polyaniline crystal structure and conductivity, Synthetic Metals, 47, (1992) pp. 187-192

[19] S. Jain, S. Chakane, A. B. Samui, V. N. Krishnamurthy and S. V. Bhoraskar, Humidity sensing with weak aciddoped polyaniline and its composites, Sensors and Actuators B: Chemical, 96, (2003) pp. 124-129

[20] K. Ogura, T. Saino, M. Nakayama and H. Shiigi, The humidity dependance of the electrical conductivity of a soluble polyaniline-poly(vinyl alcohol) composite film, Journal of Materials Chemistry, 7, (1997) pp. 2363 - 2366 [21] P. S. Barker, A. P. Monkman, M. C. Petty and R. Pride, A polyaniline/silicon hybrid field effect transistor humidity sensor, Synthetic Metals, 85, (1997) pp. 1365-1366 
[22] A. Mirmohseni, K. Valiegbal and G. G. Wallace, Preparation and characterization of a polyaniline/poly(butyl acrylate-vinyl acetate) composite as a novel conducting polymer composite. Journal of Applied Polymer Science (2003), Journal of Applied Polymer Science, 90, (2003) pp. 2525 - 2531

[23] D. Donescu, K. Gosa, A. Ciupitoiu and I. Languri, Semicontinuous emulsion polymerization of vinyl acetate. Part I. Homopolymerization with poly(vinyl alcohol) and nonionic coemulsifier, Journal of Macromolecular Science, Chemistry, A22, (1985) pp. $931-940$

[24] T. Makawinata, M. S. El-Nassr, J. W. Vanderhoff and C. Pitchot, Preparation, surface and colloidal properties of vinyl acetate-butyl acrylate copolymer latexes - effect of emulsion polymerization process and copolymer composition, Acta Polymerica, 32, (1981) pp. 583 - 592

[25] CRC Handbook of Chemistry and Physics 76th Edition, (1995 and 1996)

[26] S. Koul, S. K. Dhawan and S. Chandra, Conducting polymer polyaniline - effect of temperature on electrical conductivity transitions, 1, (1998) 332 - 334

[27] S. Kim, J. M. Ko and I. J. Chung, Electrical conductivity change of poyaniline-dodecyl benzene sulfonic acid complex with temperature, Polymers for Advanced Technologies, 7, (1996) pp. 599 - 603

[28] A. B. Kaiser, C.-J. Liu, P. W. Gilberd, B. Chapman, N. T. Kemp, B. Wessling, A. C. Partridge, W. T. Smith and J. S. Shapiro, Comparison of elctronic transport in polyaniline blends, polyaniline and polypyrrole, Synthetic Metals, 84 , (1997) pp. 699 - 702 